\title{
Time Dependence of the Cosmic Rays Positron Fraction
}

\author{
Vladimir Mikhailov ${ }^{1 *}$ \\ Emiliano Mocchiutti ${ }^{2}$ on behalf of [PAMELA collaboration] ${ }^{\dagger}$ \\ ${ }^{1}$ National Research Nuclear University MEPHI, Russia \\ ${ }^{2}$ INFN, Sezione di Trieste, Italy \\ E-mail: vvmikhajlov@mephi.ru
}

The PAMELA magnetic spectrometer is continuously gathering data about cosmic ray positrons and electrons on board the Resurs DK satellite since July 2006. Below about $10 \mathrm{GeV}$ cosmic rays are strongly modified by charge-sign dependent solar modulation effects. In this work the time variation of the positron fraction as observed by the PAMELA experiment is presented. The large data set, about nine years from 2006 till 2015, covers most of the solar cycle 24.

The 34th International Cosmic Ray Conference,

30 July- 6 August, 2015

The Hague, The Netherlands

\footnotetext{
*Speaker.

${ }^{\dagger}$ See full list of authors in attachment.
} 


\section{Introduction}

High energy electrons are produced mainly in accelerating processes in supernova remains. Small fraction of electrons are produced also in the interaction of cosmic rays with the interstellar medium together with positrons. Recent measurements of the positron to electron ratio $[1,2,3,4]$ has shown that it increases with energy beginning from $5-10 \mathrm{GeV}$, in opposition to the standard diffuse cosmic ray propagation model and interstellar mechanism of secondary production of positrons.

But also at low energy new data are clearly lower then results of previous experiments made in $90^{\text {th }}[1,3]$. Solar wind and diffusion, drift, convection processes in the Heliosperic Magnetic Field (HMF) affect cosmic-ray observations with energies less than about $10 \mathrm{GeV}$ modifying their energy spectra. This effect is called solar modulation. The effect of modulation depends on solar activity level and HMF polarity. At Solar minimum fluxes suppression is minimal and cosmic rays have maximum intensity near the Earth. It is important to note that drift process depends from sign of particles charge and lead to charge dependent modulation. Particle drift has two component related to curvature of HMF and to gradient of HMF. It also makes the heliospheric current sheet tilt angle being a very useful modulation parameter [5]. Results on the effects of the solar modulation on the energy spectra of galactic cosmic-ray protons for period between June 2006 and December 2009 have already been published [6]. Here results on the long-term variation in the positron fraction of galactic cosmic-ray are presented at $1 \mathrm{AU}$. These results are based on the data set collected by the PAMELA (Payload for Antimatter Matter Exploration and Light-nuclei Astrophysics) satelliteborne experiment. PAMELA is an instrument designed for cosmic-ray antimatter studies and is flying on-board the Russian Resurs-DK1 satellite.

\section{The experiment}

The PAMELA magnetic spectrometer is mounted on the Resurs-DK1 satellite, which was placed into near Earth elliptical orbit with an altitude of $350-600 \mathrm{~km}$ and an inclination of $70.4^{\circ}$ on June 15, 2006. The spectrometer includes several detector systems capable of measuring particle speed $\beta$, charge $Z$, rigidity $R$ and total energy $E$. The electron and positron identification is provided by the imaging calorimeter which consists of a set of strip silicon detectors interleaved by tungsten planes (16.3 radiation and 0.6 nuclear interaction lengths deep). The instrument allows extracting electrons and positrons and measuring their energy from $50 \mathrm{MeV}$ to several hundred $\mathrm{GeV}$. Electrons and positrons were identified with using information from the magnetic spectrometer electromagnetic calorimeter, time-of flight system, neutron detector and anticoincidence system. Details of the experiment and of its particle identifications can be found in[1, 6].

\section{Data Analysis}

In this paper the analysis based on data gathered from June 2006 to February 2014. Data till June 2015 will be presented at the conference.

Total accumulated statistic for electrons and positron is about $4 \times 10^{6}$ events with energy more then $100 \mathrm{MeV}$. 
Figure 1 shows heliospheric condition before and during measurements. F10.7 cm flux is perfect index for solar activity. PAMELA started observations at the end of $23^{\text {th }}$ solar cycle. Solar activity was decreasing at the beginning of the experiment in 2006 and it reached the minimum in the 2009. The highest proton flux was observed by PAMELA at the end of 2009 [6].

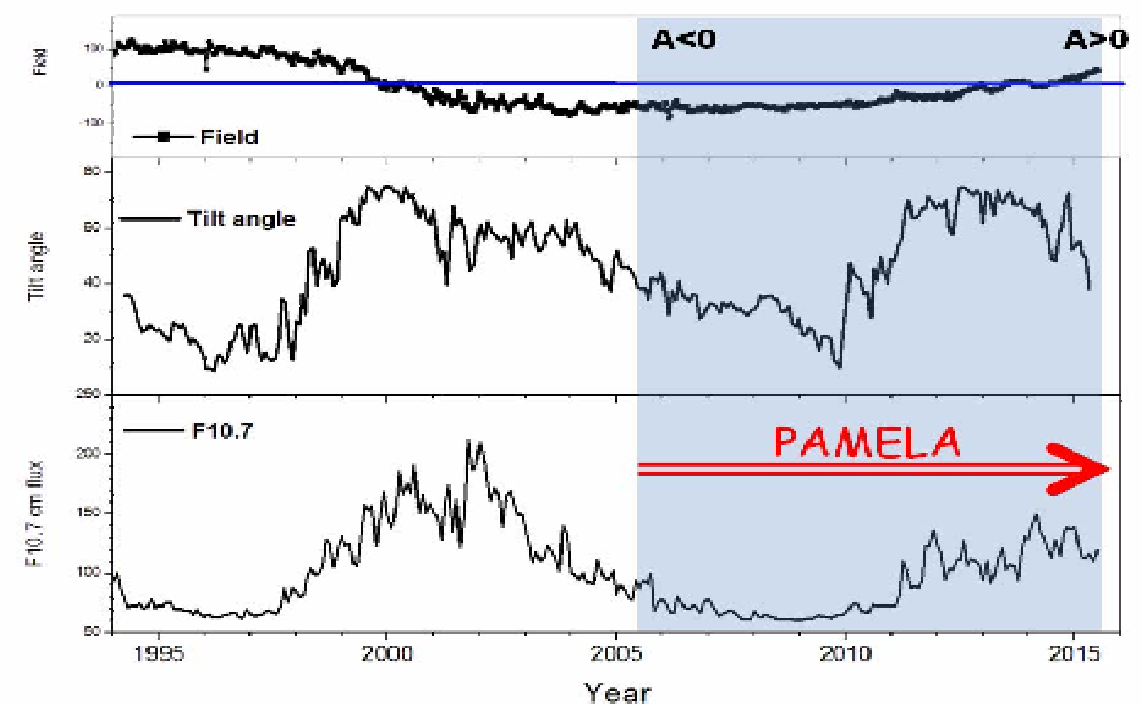

Figure 1: PAMELA observations are related to different periods of solar activity. Top panel shows projection of heliosperic magnetic field, middle panel shows tilt angle (data of the Wilcox Solar Observatory, http://wso.stanford.edu ) and bottom panel shows F10.7 cm flux (http://www.spaceweather.gc.ca/solarflux/sx-5-mavg-en.php)

Tilt angle is important parameter to characterize drift of particles along of neutral sheet of HMF. As it is clear from figure 1, the drift was maximal in 2009. Polarity of HMF changed from positive sign $\mathrm{A}>0$ to negative $\mathrm{A}<0$ in 2002 and in 2014 it changed again.

Using a geographical coordinates the McIlvain geomagnetic coordinates L-shell and B were calculated for each event. For the calculation, the IGRF model [7] of the Earth magnetic field was used. Primary cosmic ray particles were selected by vertical geomagnetic rigidity cut-off $\mathbf{R}_{\text {cut }}$ in point of observation requiring particle rigidity $\mathrm{R}>1.2 \times \mathrm{R}_{\text {cut }}$.

\section{Results and Discussion}

The PAMELA observations covers period from the end of last long $23^{\text {rd }}$ solar minimum in 2006 till the maximum of next $24^{\text {th }}$ cycle including the polarity change interval from $\mathrm{A}<0$ to $\mathrm{A}>0$. Figure 2 shows measured positron fraction at average energy $\langle\mathrm{E}\rangle=1.2 \mathrm{GeV}$ as function of time. High statistical precision of data allow us to limit the role of statistical errors and to see the variations of ratio even if they are relatively small in period before 2014. While large solar modulation of electrons, positron and proton fluxes was observed, variation of positron fraction were relatively small. Maximum of the ratio was reached at the end of 2009, at minimum of solar activity, because of more fast increasing of the positron flux comparing to electron at that period. 


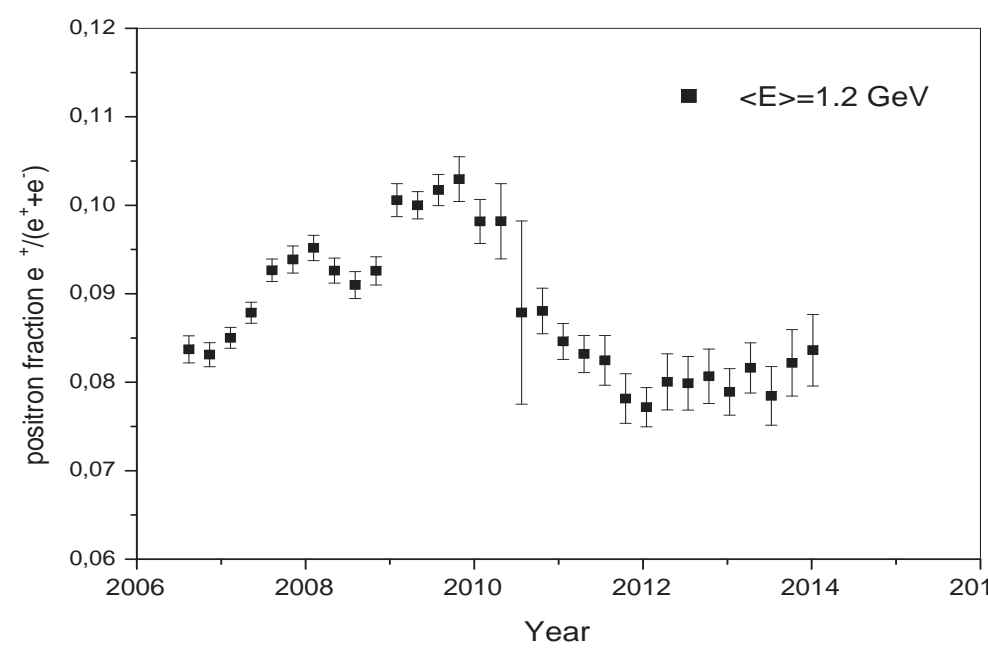

Figure 2: Cosmic ray positron to electron ratio vs time, $\langle\mathrm{E}\rangle=1.2 \mathrm{GeV}$.

Several attempts were made to estimate electron and positron fluxes variations during solar cycle. All models show the different behavior occurring in periods with different magnetic field polarity. During periods with $\mathrm{A}>0$ the positrons ratio is quite similar to the interstellar model. During periods with opposite field polarity instead there is a relevant reduction of the cosmic ray positron fraction in comparison with the interstellar one [5, 8]. Meanwhile, quantitative agreement with observations is less perfect. For example, the results of PAMELA spectrometer shows that the modeled magnitude [9] is somewhat too large during whole solar cycle.

\section{Summary}

Measured in the PAMELA experiment electron -positron fluxes and their ratios due to large accumulated statistics allow to detail study modulation effects during $9^{\text {th }}$ year period.

\section{Acknowlegments}

We acknowledge support from the Italian Space Agency (ASI), Russian Federal Space Agency, Deutsches Zentrum fur Luft- und Raumfahrt (DLR), the Swedish National Space Board, the Swedish Research Council. Russian colleagues also acknowledge partial support from Russian Scientific Foundation (grant 14-12-00373).

\section{References}

[1] Adriani O., Barbarino G. C,. Bazilevskaya G. A. et al. [PAMELA collaboration] //Nature. 2009. V.458. P. 607.

[2] Ackermann M., Ajello M., Atwood W. et al. [Fermi collaboration] //Phys. Rev. D. 2011. V.84. P. 032007. 
[3] Aguilar M., Alberti G., Alpat B. et al. [AMS collaboration ] //Phys. Rev. Lett. 2013. V. 96. P. 161-172.

[4] Accardo L.,Aguilar M., Aisa D., et al. [AMS collaboration ] //Phys. Rev. Lett. 2014. V. 113. P.121101.

[5] Potgieter M. //Advances in Space Research. 2014. V.53 P.1415-1425.

[6] Adriani O., Barbarino G. C,. Bazilevskaya G. A. et al. [PAMELA collaboration] //Astrophysical Journal. 2013. V.765. P.91. doi:10.1029/2009JA014660.

[7] http://nssdcftp.gsfc.nasa.gov/models/geomagnetic/igrf

[8] Della Torre S., Bobik P., Boschini M.J. et al.//Advances in Space Research. 2012. V.49 P.1587Ü1592.

[9] Clem J.M, Clements D.P., Esposito J. et al.//Astrophysical Journal. 1996. V.464. P.507-515. 